\title{
Hypoxia Modulates Platelet Purinergic Signalling Pathways
}

\author{
Gordon G. Paterson ${ }^{1,2}$ Jason M. Young ${ }^{1,2}$ Joseph A. Willson ${ }^{3}$ Christopher J. Graham ${ }^{1,2}$ \\ Rebecca C. Dru ${ }^{1,2}$ Eleanor W. Lee ${ }^{1,2}$ Greig S. Torpey ${ }^{1,2}$ Sarah R. Walmsley ${ }^{3}$ Melissa V. Chan ${ }^{4}$ \\ Timothy D. Warner ${ }^{4}$ John Kenneth Baillie ${ }^{1,5,6}$ Alfred Arthur Roger Thompson ${ }^{1,70}$
}

\footnotetext{
${ }^{1}$ APEX (Altitude Physiology Expeditions), Edinburgh, United Kingdom

2 Edinburgh Medical School, University of Edinburgh, Edinburgh, United Kingdom

3 University of Edinburgh Centre for Inflammation Research, The Queen's Medical Research Institute, University of Edinburgh, Edinburgh, United Kingdom

${ }^{4}$ Centre for Immunobiology, Blizard Institute, Barts and The London School of Medicine and Dentistry, Queen Mary University of London, London, United Kingdom

${ }^{5}$ Division of Genetics and Genomics, The Roslin Institute, University of Edinburgh, Edinburgh, United Kingdom

${ }^{6}$ Department of Anaesthesia, Critical Care and Pain Medicine, Royal Infirmary of Edinburgh, NHS Lothian, Edinburgh, United Kingdom

${ }^{7}$ Department of Infection, Immunity and Cardiovascular Disease, University of Sheffield, Sheffield, United Kingdom
}

Address for correspondence Alfred Arthur Roger Thompson, BSc, MB ChB, MRCP, PhD, Department of Infection, Immunity and Cardiovascular Disease, University of Sheffield, M127a, Royal Hallamshire Hospital, Beech Hill Road, Sheffield S10 2RX, United Kingdom (e-mail: R.Thompson@sheffield.ac.uk).

Thromb Haemost 2020;120:253-261.

\begin{abstract}
Keywords

- hypoxia

- platelet physiology

- anti-platelet agents

- ADP receptors

- high altitude

Background Hypoxia resulting from ascent to high-altitude or pathological states at sea level is known to increase platelet reactivity. Previous work from our group has suggested that this may be adenosine diphosphate (ADP)-specific. Given the clinical importance of drugs targeting ADP pathways, research into the impact of hypoxia on platelet ADP pathways is highly important.

Methods Optimul aggregometry was performed on plasma from 29 lowland residents ascending to $4,700 \mathrm{~m}$, allowing systematic assessment of platelet reactivity in response to several platelet agonists. Aggregometry was also performed in response to ADP in the presence of inhibitors of the two main ADP receptors, $\mathrm{P}_{2} \mathrm{Y}_{1}$ and $\mathrm{P}_{2 \mathrm{Y}_{12}}$ (MRS2500 and cangrelor, respectively). Phosphorylation of vasodilator-stimulated phosphoprotein (VASP), a key determinant of platelet aggregation, was analysed using the VASPFix assay. Results Hypobaric hypoxia significantly reduced the ability of a fixed concentration of cangrelor to inhibit ADP-induced aggregation and increased basal VASP phosphorylation. However, in the absence of P2Y receptor inhibitors, we did not find evidence of increased platelet sensitivity to any of the agonists tested and found reduced sensitivity to thrombin receptor-activating peptide- 6 amide.

Conclusion Our results provide evidence of increased $\mathrm{P}_{2} \mathrm{Y}_{1}$ receptor activity at high altitude and suggest down-regulation of the $\mathrm{P} 2 \mathrm{Y}_{12}$ pathway through increased VASP phosphorylation. These changes in ADP pathway activity are of potential therapeutic significance to high-altitude sojourners and hypoxic sea level patients prescribed platelet inhibitors and warrant further investigation.
\end{abstract}

received

April 24, 2019

accepted after revision

September 29, 2019
DOI https://doi.org/

$10.1055 / \mathrm{s}-0039-3400305$.

ISSN 0340-6245.
(C) 2020 Georg Thieme Verlag KG Stuttgart . New York
License terms

(c) (i) 


\section{Introduction}

Acute hypobaric hypoxia, such as that induced by ascent to high altitude, has long been considered to produce a thrombogenic phenotype. ${ }^{1}$ Consistent with this, epidemiological studies report a markedly increased risk of strokes at high altitude: up to 30 times that at sea level. ${ }^{2}$ Furthermore, these events are reported to occur in younger patients with fewer cardiovascular risk factors. ${ }^{3}$ Although some studies examining the effect of acute hypoxia on coagulation have reported minimal changes in coagulation, they were investigating the 'economy class syndrome' and thus exposure to hypoxia was brief. ${ }^{4,5}$ On the contrary, studies examining a longer exposure to hypoxia have demonstrated a hypercoagulable state. ${ }^{6-8}$

Platelets are key to haemostasis ${ }^{9,10}$ and appear to have an important role in the hypercoagulable state induced by hypoxia: expression of soluble P-selectin, an in vivo platelet activation marker, was shown to be increased 2.5 -fold after ascent to high altitude. ${ }^{11}$ Furthermore, hypoxia has recently been shown to significantly alter the platelet proteome, including up-regulation of calpain small subunit $1 .^{12}$ Calpains are calcium-dependent proteases involved in various physiological processes including platelet activation. ${ }^{13}$ The same study also found increased intracellular calcium concentration in hypoxic platelets $^{12}$ which is a common downstream effect of platelet activation by several agonists, including adenosine diphosphate (ADP). ${ }^{10}$

Recent work from our group has demonstrated a hypercoagulable state in 63 subjects participating in a controlled, nonexertional sojourn to $5,300 \mathrm{~m} .{ }^{6}$ Multiplate aggregation assays using fixed doses of several platelet activators at this altitude suggested that hypoxia-induced platelet hyper-reactivity is specific to ADP as responses to thrombin receptor-activating peptide (TRAP) and collagen were not altered. ${ }^{6}$ It is known that ADP acts on two pro-aggregatory pathways, via $\mathrm{P} 2 \mathrm{Y}_{1}$ and $\mathrm{P} 2 \mathrm{Y}_{12}$ receptors, ${ }^{14}$ and in light of our previous findings we aimed to further investigate these pathways. $\mathrm{P}_{2} \mathrm{Y}_{12}$ inhibitors are widely used anti-platelet medications, and effects of hypoxia on their efficacy are of high clinical relevance. This is particularly important given the increasing number of older sojourners to high altitude, who may have cardiovascular co-morbidities. ${ }^{15}$

Our study is the first to our knowledge to investigate the effect of hypoxia on the major platelet activation pathways with full concentration-response curves. Optimul aggregometry is a recently developed assay which applies the principles of light-transmission aggregometry to a 96-well format, with platelet agonists lyophilised in each well. ${ }^{16}$ This assay requires significantly less plasma volume than traditional aggregometry, thus facilitating systematic assessment of platelet aggregation in response to several agonists. Ex vivo use of ADP receptor inhibitors also permitted further detailed study of the purinergic pathways in a group of healthy, lowland volunteers ascending to $4,700 \mathrm{~m}$.

\section{Methods}

Data were collected from 29 participants before and during the APEX 5 Expedition. All participants had no known cardiovascular or respiratory conditions and no pre-existing coagulopathy. Participants were asked to refrain from alcohol and anti-platelet medications during the week prior to sampling. This study was approved by the ACCORD Research Ethics Committee (17-HV-030) and all participants gave informed consent as per the Declaration of Helsinki. Reagents were supplied by Sigma-Aldrich (Irvine, United Kingdom) unless otherwise specified.

\section{Ascent Profile and Sample Collection}

Participants were resident at $<250 \mathrm{~m}$ above sea level and had not travelled to high altitude $(>2,500 \mathrm{~m})$ in the 2 months prior to the study. The ascent profile and study timeline are summarised in -Fig. 1. We chose to perform aggregometry assays on day 6 (one day following ascent to $4,700 \mathrm{~m}$ to examine effects of sub-acute hypoxia) and day 11 (the time point we had previously observed changes in Multiplate aggregation with $\mathrm{ADP}^{6}$ ). Venepuncture was performed using $21 \mathrm{G}$ needles (Williams Medical, Rhymney, United Kingdom) into citrated tubes. Platelet-rich plasma (PRP) was prepared by centrifugation of whole blood at $175 \times g$ for 15 minutes (EBA 280, Hettich, Tuttlingen, Germany). Platelet-poor plasma (PPP) was generated by further centrifugation at $3,624 \times g$ for 2 minutes. Peripheral oxygen saturation $\left(\mathrm{SpO}_{2}\right)$ was measured using a pulse oximeter (SM-100, Santamedical, Tustin, United States) at baseline and every day of the expedition.

\section{Optimul Aggregometry}

Modified optimul aggregometry plates were prepared as previously described by Chan and Warner. ${ }^{16}$ In brief, prediluted platelet agonists were added to individual wells of a gelatin-coated 96-well plate and the plates were lyophilised. Plates were vacuum-sealed and protected from light before use. Each 96-well plate contained lyophilised concentration ranges of arachidonic acid (0.03-1 mM), ADP (0.005-40 $\mu \mathrm{M})$,

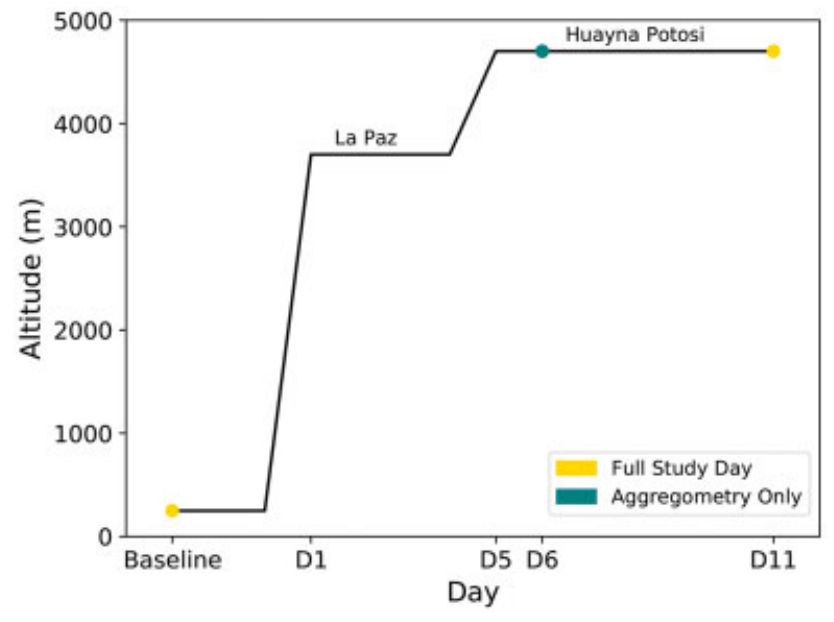

Fig. 1 Ascent profile. Baseline testing was performed in April 2017, 2 months prior to the expedition. Subjects landed in La Paz (3,700 m) where they spent four nights before ascending to Huayna Potosi Base Camp $(4,700 \mathrm{~m})$ by bus on day 5 where they stayed for the remainder of the study. Optimul aggregometry, VASPFix and full blood count samples were collected at baseline and on day 11 . On day 6 , only optimul aggregometry was performed. 
collagen $(0.01-40 \mu \mathrm{g} / \mathrm{mL})$, epinephrine $(0.0004-10 \mu \mathrm{M})$, TRAP-6 amide $(0.03-40 \mu \mathrm{M})$, and U46619 (thromboxane mimetic, $0.005-40 \mu \mathrm{M}$ ).

PRP was aliquoted and incubated for 30 minutes (at $37^{\circ} \mathrm{C}$ ) alone or in the presence of the $\mathrm{P}_{2} \mathrm{Y}_{1}$ inhibitor MRS2500 $(1 \mu \mathrm{M}$, Tocris Bioscience, Bristol, United Kingdom) or the $\mathrm{P}_{2} \mathrm{Y}_{12}$ inhibitor cangrelor $(100 \mathrm{nM}$, The Medicines Company, New Jersey, United States). After incubation, $40 \mu \mathrm{L}$ of PRP (plus or minus inhibitor) was quickly added to wells containing the lyophilised platelet agonists. PPP was added to four agonist-free wells to provide a signal equivalent to $100 \%$ aggregation. PRP was also added to agonist-free wells and wells containing vehicle alone as a $0 \%$ aggregation control. Plates were then placed on a thermal shaker (BioShake iQ QInstruments, Jena, Germany) for 5 minutes $\left(37^{\circ} \mathrm{C}, 1,200\right.$ revolutions per minute). Light absorbance was read at $595 \mathrm{~nm}$ in a 96-well plate reader (SPECTROstar Nano, BMG LABTECH, Aylesbury, United Kingdom).

Percentage aggregation was calculated using absorbance values for PPP (100\%) and PRP (0\%) in agonist-free wells as reference values. Avisual inspection of concentration-response curves was conducted to remove any clearly erroneous curves (deviating from a sigmoid shape). Data were also removed if they showed no response, with response defined as greater than 30\% aggregation in response to two doses of agonist.

\section{Vasodilator-Stimulated Phosphoprotein Phosphorylation}

Vasodilator-stimulated phosphoprotein (VASP) is a platelet protein whose phosphorylation is modulated by cyclic adenosine monophosphate (CAMP). ${ }^{17}$ cAMP production is inhibited by the action of $\mathrm{G}_{\alpha \mathrm{i}}$ on adenylate cyclase secondary to activation of P2Y ${ }_{12}$ receptors. ${ }^{18}$ The degree of VASP phosphorylation can thus be used as a marker of P2Y $_{12}$ activity. ${ }^{19}$ The VASPFix assay ${ }^{20}$ was used to quantify VASP phosphorylation.

Aliquots of PRP were incubated for 6 minutes in one of three conditions: phosphate-buffered saline (PBS), a prostacyclin analogue iloprost $(1 \mathrm{nM})$, or ADP $(5 \mu \mathrm{M})+$ iloprost $(1 \mathrm{nM})$ and mixed in a 1:5 ratio with VASPFix (Platelet Solutions Ltd., Nottingham, United Kingdom), vortexed, and snap frozen on dry ice. Samples from high altitude were transported to the United Kingdom for analysis on dry ice by a specialist company.

Flow cytometry was performed and the median fluorescein isothiocyanate fluorescence ( $\mathrm{mf}$ ) recorded for each condition. Iloprost induces maximal VASP phosphorylation with PBS acting as a negative control.

If iloprost did not induce phosphorylation (i.e., if $\mathrm{mf}$ (iloprost $)<\operatorname{mf}($ saline)), it was assumed that there was a technical failure and these samples removed from the analysis. Similarly, if $\mathrm{mf}$ (iloprost + ADP) was greater than $\mathrm{mf}$ (iloprost), the sample was also removed from analysis.

\section{Full Blood Count}

Three millilitres of blood were collected into an ethylenediaminetetraacetic acid blood tube (Sarstedt Ltd., Leicester, United Kingdom) and samples analysed within 24 hours by clinical haematology laboratories (NHS Lothian Laboratories, Edin- burgh, United Kingdom, and SELADIS, Universidad Mayor de San Andrés, La Paz, Bolivia).

\section{Statistics}

Optimul aggregometry data were fit to Eq. (1) by least squares, non-linear regression using the scipy.optimize library (for Python 2.7.2).

Eq. (1): Agonist-response equation where $y$ is the percentage aggregation, $x$ is $\log$ [agonist], $\mathrm{EC}_{50}$ the concentration resulting in half maximal aggregation, Top/Bottom the maximum/minimum aggregation and the Hill Slope the steepness of the curve. For studies examining the dose-response curve of cangrelor, $\mathrm{IC}_{50}$ replaces $\mathrm{EC}_{50}$.

$$
y=\frac{\text { Bottom }+(\text { Top }- \text { Bottom })}{1+10^{(\text {LogEC50-x) } \cdot \text { Hill Slope }}}
$$

The concentration resulting in half-maximal aggregation $\left(\mathrm{EC}_{50}\right)$ was used to compare curves from each time point. For comparisons between ADP concentration-response curves in the presence of a fixed antagonist, maximum response $\left(R_{\max }\right)$ to ADP was also calculated.

Outlier identification was based on difference of fits analysis. ${ }^{21}$ The effect of each point on $\mathrm{EC}_{50}\left(\Delta \mathrm{EC}_{50}\right)$ and Hill Slope $(\Delta$ Hill) was calculated by iterative removal of points and refitting the curves. $\triangle \mathrm{EC}_{50}$ and $\Delta$ Hill values were reviewed for comparable data (all points in all concentration-response curves for one agonist at one time point) and any points falling out with two standard deviations $\left(\Delta \mathrm{EC}_{50}\right)$ or four standard deviations ( $\Delta$ Hill) were considered outliers. Additionally, for concentration-response curves in the presence of inhibitors, if the final point was less than $75 \%$ of the point preceding it, it too was considered an outlier, to ensure validity of the $R_{\max }$ parameter.

Wherever possible, paired statistics were used; however, the optimul aggregometry data were unpaired. For paired comparisons of two time points, paired Student's t-tests were performed using the scipy.stats library. ${ }^{22}$ For paired data compared over three time points $\left(\mathrm{SpO}_{2}\right)$, a one-way repeated-measure analysis of variance (ANOVA) was conducted with a Tukey's HSD post hoc test using Prism 5.0 (GraphPad, La Jolla, California, United States). For optimul aggregometry data, comparisons between three time points were analysed using one-way ANOVA with Tukey's HSD post hoc test using R 3.4.2. ${ }^{23}$ Whenever multiple comparisons were made, between multiple agonists or conditions, $p$-values reported were adjusted using a Bonferroni correction. Statistical significance was set as 0.05 .

\section{Results}

Baseline characteristics of subjects are summarised in - Table 1.

All but one subject completed the study, and data have been included until dropout for unpaired analyses. The sojourn at high altitude induced a marked hypoxaemia (-Fig. 2A), which was slightly more pronounced on day 11 than day 6 . Platelet counts were also significantly elevated by 
Table 1 Baseline characteristics

\begin{tabular}{|l|l|}
\hline Characteristic & APEX 5 cohort \\
\hline Sex (male/female) & $9 / 20$ \\
\hline Mean BMI (range) & $22.3(17.6-28.8)$ \\
\hline Mean age (range) & $20.7(18-26)$ \\
\hline
\end{tabular}

Note: Body mass index (BMI) has units $\mathrm{kg} / \mathrm{m}^{2}$, and age is measured in years.
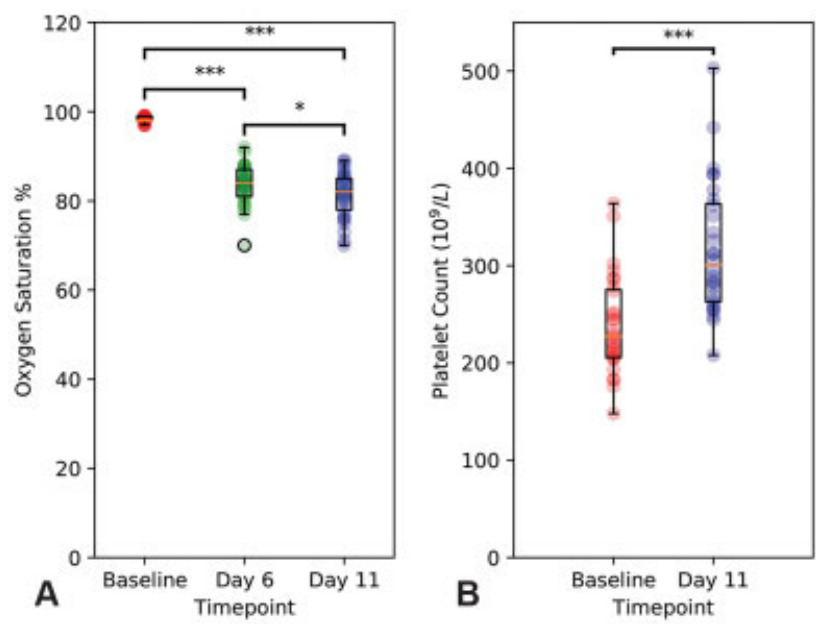

Fig. 2 Platelet counts and oxygen saturations. (A) Oxygen saturation was measured at baseline and on day 6 and day 11 of the expedition. Data points are represented as semi-translucent circles, with summary box plots superimposed. Data were analysed by a one-way repeated-measure analysis of variance (ANOVA) followed by a Tukey's HSD test. (B) Platelet count was measured at baseline and on day 11 of the expedition. Data points are represented as semi-translucent circles, with summary box plots superimposed. Data were analysed by paired Student's t-test. ${ }^{* * *} p<0.001,{ }^{*} p<0.05$.

hypoxia ( - Fig. 2B). Haemoglobin, haematocrit and white cell count data are provided in - Supplementary Table $\mathbf{S 1}$ (available in the online version).

Hypoxic Platelets are Less Sensitive to TRAP-6 Amide The effect of hypoxia on various platelet agonists was compared between baseline, day 6 and day 11. No platelet activation pathways were found to be sensitised by hypoxia; however, platelets became less sensitive to TRAP-6 amide ( - Fig. 3E, - Table $2, p<0.01$ ). This effect was only present on day 11 .

\section{Hypoxia Modulates Purinergic Signalling}

In the presence of $1 \mu \mathrm{M} \mathrm{P2Y}$ inhibitor MRS2500, both $\mathrm{EC}_{50}$ and $R_{\max }$ were unchanged by hypoxia ( $\mathbf{F i g . ~ 4 A , ~ - ~ T a b l e ~} 2$ ). Likewise, in the presence of $100 \mathrm{nM} \mathrm{P2Y_{12 }}$ inhibitor cangrelor, $\mathrm{EC}_{50}$ remained unchanged after 11 days of hypoxia. While $\mathrm{EC}_{50}$ remained unchanged at altitude, $R_{\max }$ was markedly increased on both days 6 and 11 compared with baseline (-Fig. 4B, - Table 2, $p<0.01$ ).

\section{Hypoxia Modulates Basal VASP Phosphorylation in Platelets}

Compared with baseline, after 11 days at high altitude there was a highly significant increased $\mathrm{mf}$ in the PBS-treated samples ( - Fig. 5A). There were no differences between baseline and day 11 in other conditions (-Fig. 5A). There was a marked reduction in the $\mathrm{mf}$ percentage increase induced by iloprost at high altitude (-Fig. 5B), but no change in $\mathrm{mf}$ reduction induced by the addition of $\mathrm{ADP}$ ( - Fig. 5C).

\section{Discussion}

There is a growing body of evidence that hypoxia induces hyper-reactivity in platelets, a phenomenon important to both altitude physiology and sea-level pathophysiology. We found that hypoxia increases maximal aggregation to ADP in the presence of cangrelor and down-regulates platelet sensitivity to TRAP-6 amide. However, we did not demonstrate sensitisation to any of the platelet activators tested. Finally, and more strikingly, we have demonstrated modulation of basal VASP phosphorylation, a key step in the $\mathrm{P}_{2} \mathrm{Y}_{12}$ signalling pathway.

Our results contrast with a previous study from our group which reported increased platelet sensitivity to ADP. ${ }^{6}$ Our previous findings were based on Multiplate analysis and discrepancies could be related to the impact of platelet count on the two different assays: Multiplate is more sensitive to platelet count than optimul aggregometry. ${ }^{24,25}$ This, however, would not explain why our previous findings were specific to ADP. Perhaps more likely is that differences relate to the parameter of platelet function that was tested. Multiplate is based on electrical-impedance aggregometry measured in real-time over 6 minutes, with the area under the impedance time curve as the final outcome. ${ }^{26}$ This outcome is therefore dependent on both the final amplitude of the response and the rate at which this is reached. Optimul aggregometry, however, is based on one measurement at 5 minutes. Conceivably, differences in the ADP sensitivity could be time dependent, given the time sensitivity of phosphorylation ${ }^{27}$ and changes in purinergic receptor expression ${ }^{28}$ induced by ADP stimulation.

While we have not resolved the uncertainty surrounding ADP sensitivity, our study did reveal interesting changes in purinergic signalling. The $R_{\max }$ induced by ADP in the presence of $100 \mathrm{nM}$ cangrelor was significantly higher at altitude (-Fig. 4B, - Table 2). This could be due to increased $\mathrm{P}_{2} \mathrm{Y}_{1}$ receptor activity. An increase in $\mathrm{P}_{2} \mathrm{Y}_{1}$ activity may imply a difference in receptor expression or modulation of receptor activity since G-protein-coupled receptor activity can be regulated by multiple mechanisms. ${ }^{29}$ Increased $\mathrm{P}_{2} \mathrm{Y}_{1}$ activity would provide an interesting link to the proteomic data of Tyagi et al who described increased calpain expression and activity in rats exposed to hypoxia for 6 hours, ${ }^{12}$ since a downstream consequence of $\mathrm{P}_{2} \mathrm{Y}_{1}$ activation is increased intracellular calcium. ${ }^{30}$

Well-controlled, high-altitude expeditions are excellent models of acute hypoxia ${ }^{31}$ and their findings are often relevant to hypoxic patients at sea level. In this regard, patients with chronic hypoxic diseases such as chronic obstructive pulmonary disease and obstructive sleep apnoea (OSA) have an increased risk of thrombotic events such as myocardial infarction and stroke. ${ }^{32,33}$ Furthermore, these conditions are also associated with increased platelet reactivity, which in the case of OSA is reversed on correction of hypoxia. ${ }^{34-36}$ However, the 

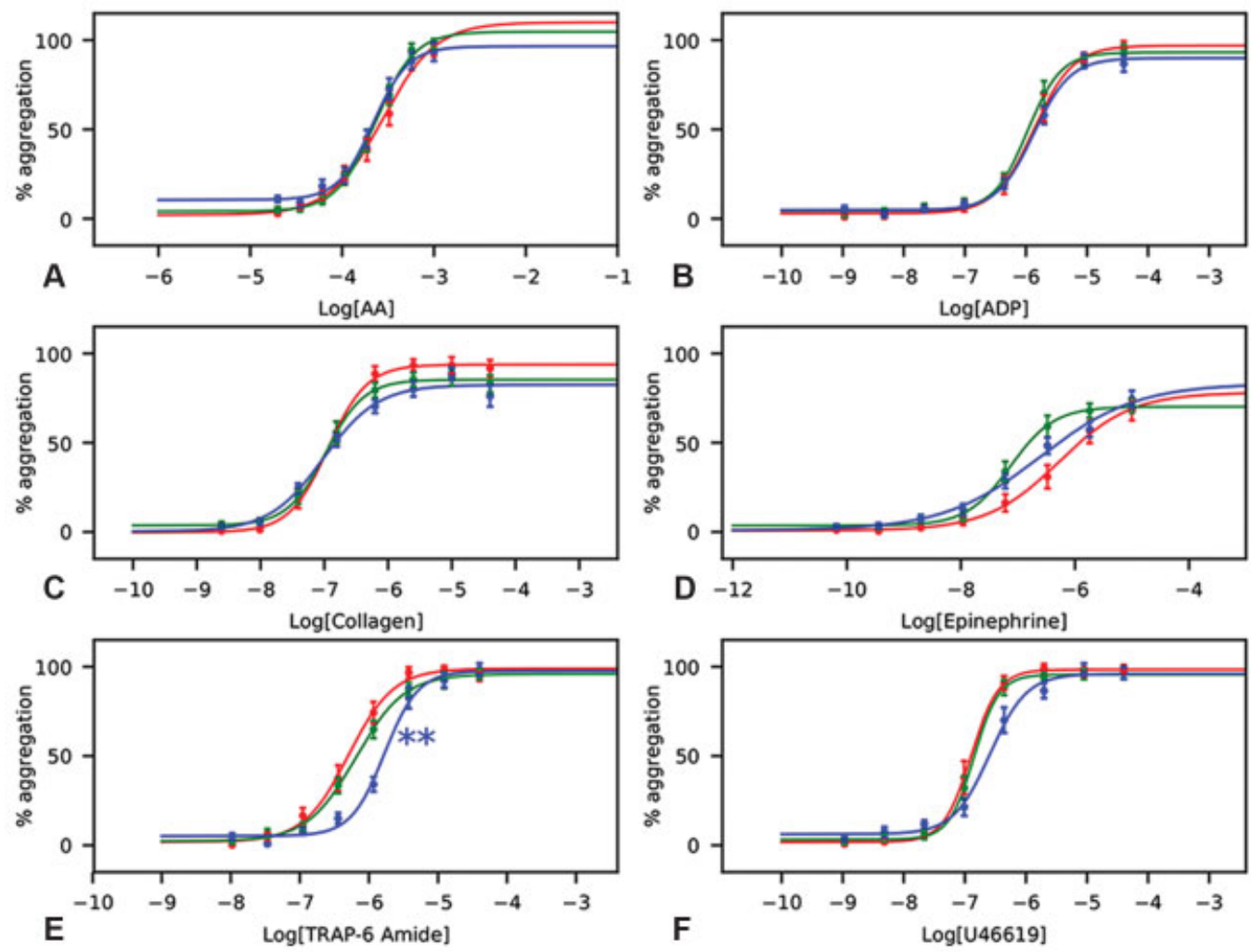

Fig. 3 The effect of hypoxia on platelet activation pathways. Dose-response curves of platelet aggregation in response to (A) arachidonic acid (AA), (B) adenosine diphosphate (ADP), (C) collagen, (D) epinephrine, (E) thrombin receptor-activating peptide (TRAP)-6 amide and (F) U46619. Data are mean percentage aggregation \pm standard error of the mean (SEM), and best fit curves optimised to these mean values. $E_{50} s$ were compared by one-way analysis of variance (ANOVA) followed by Tukey's HSD post hoc tests where appropriate. $p$-Values reported were adjusted to account for family-wise error rate using Bonferroni corrections. ${ }^{* *} \mathrm{p}<0.01$ vs. baseline.

Table 2 Summary results of optimul aggregometry data

\begin{tabular}{|c|c|c|c|}
\hline \multicolumn{4}{|l|}{$\mathrm{EC}_{50}$ values } \\
\hline & Baseline & Day 6 & Day 11 \\
\hline AA & $-3.695(0.053)$ & $-3.696(0.039)$ & $-3.715(0.064)$ \\
\hline ADP & $-5.990(0.096)$ & $-6.037(0.122)$ & $-5.912(0.078)$ \\
\hline Collagen & $-6.776(0.111)$ & $-7.009(0.107)$ & $-6.853(0.088)$ \\
\hline Epinephrine & $-6.339(0.194)$ & $-6.805(0.144)$ & $-6.584(0.140)$ \\
\hline TRAP-6 amide & $-6.314(0.105)$ & $-6.221(0.064)$ & $-5.759(0.084)^{a}$ \\
\hline U46619 & $-6.979(0.122)$ & $-6.652(0.099)$ & $-6.545(0.122)$ \\
\hline ADP + MRS2500 & $-5.280(0.082)$ & $-5.222(0.063)$ & $-5.244(0.064)$ \\
\hline ADP + Cangrelor & $-5.186(0.062)$ & $-5.318(0.068)$ & $-5.335(0.079)$ \\
\hline \multicolumn{4}{|l|}{$R_{\max }$ values } \\
\hline$A D P+M R S 2500$ & $95.230(2.835)$ & $100.413(2.159)$ & $96.638(2.788)$ \\
\hline ADP + Cangrelor & $80.005(4.177)$ & $97.927(2.557)^{\mathrm{a}}$ & $97.186(2.398)^{a}$ \\
\hline
\end{tabular}

Abbreviations: AA, arachidonic acid; ADP, adenosine diphosphate; PRP, platelet-rich plasma; TRAP, thrombin receptor-activating peptide.

Note: Dose-response curves of platelet aggregation were performed in response to AA, ADP, collagen, epinephrine, TRAP-6 amide, and U46619. Aggregometry was also performed on PRP incubated for 30 minutes with $1 \mu \mathrm{M}$ MRS2500 (P2 $\mathrm{Y}_{1}$ inhibitor) or $100 \mathrm{nM}$ cangrelor ( $\left(\mathrm{P} 2 \mathrm{Y}_{12}\right.$ inhibitor) with $\mathrm{ADP}$ as the agonist. For experiments with inhibitors, the maximal response $\left(R_{\max }\right)$ was also calculated. $\mathrm{EC}_{50}$ and $R_{\max }$ values were compared by oneway analysis of variance (ANOVA) followed by Tukey's HSD post hoc tests where appropriate. $p$-Values reported were adjusted to account for familywise error rate using Bonferroni corrections.

${ }^{\mathrm{a}} \mathrm{p}<0.01$ vs. baseline. 

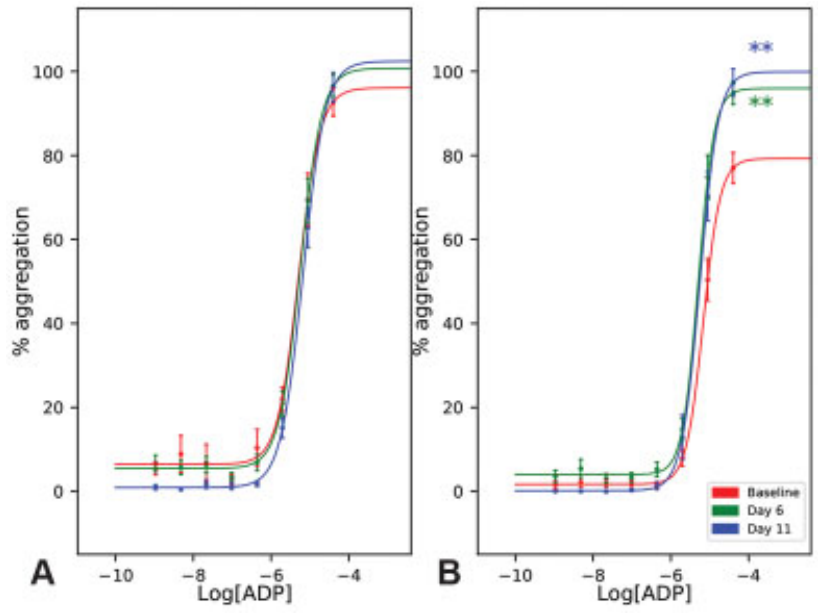

Fig. 4 The effect of hypoxia on adenosine diphosphate (ADP)-induced platelet aggregation in the presence of fixed doses of inhibitors. Platelet-rich plasma (PRP) was incubated for 30 minutes with (A) $1 \mu \mathrm{M}$ MRS2500 (P2Y 1 inhibitor) or (B) $100 \mathrm{nM}$ cangrelor (P2Y 12 inhibitor). Data are mean percentage aggregation \pm standard error of the mean (SEM), and best fit curves optimised to these mean values. $\mathrm{EC}_{50}$ and $R_{\max }$ values were compared by one-way analysis of variance (ANOVA) followed by Tukey's HSD post hoc tests where appropriate. $p$-Values reported were adjusted to account for family-wise error rate using Bonferroni corrections. ${ }^{* *} p<0.01$ vs. baseline.

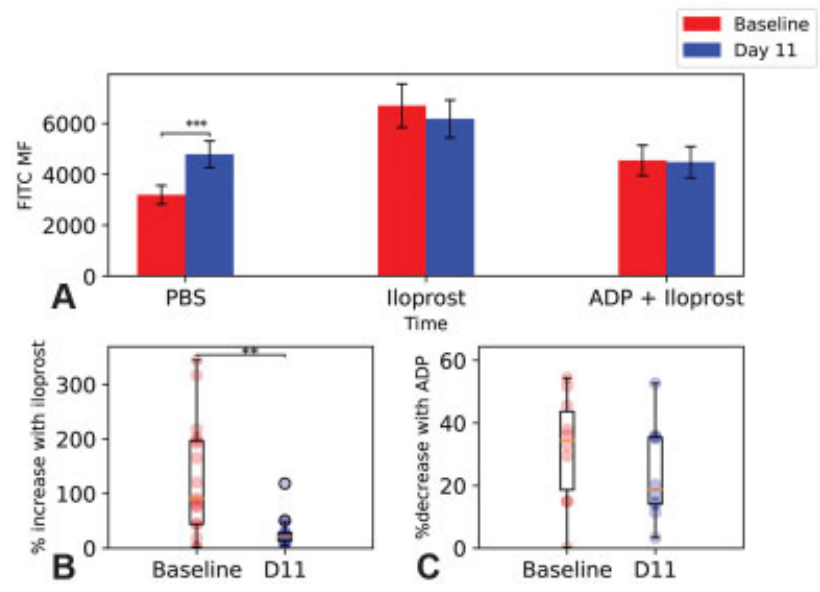

Fig. 5 The effect of hypoxia on platelet vasodilator-stimulated phosphoprotein (VASP) phosphorylation. Platelet-rich plasma (PRP) was incubated for 6 minutes with phosphate-buffered saline (PBS), iloprost or adenosine diphosphate (ADP) + lloprost before addition of VASPFix. Flow cytometry was used to identify the fluorescein isothiocyanate (FITC) median fluorescence (MF), reflecting the degree of VASP phosphorylation. (A) Raw FITC MF for each condition at each time point. Data are mean FITC MF \pm standard error of the mean (SEM). Data were compared by paired $t$-tests with $p$-values adjusted by Bonferroni correction. (B) Percentage increase in FITC MF induced by addition of iloprost. (C) Percentage decrease induced by ADP addition. (B, C) Individual data points are represented by semi-translucent circles with box plots superimposed. Data were compared by paired $t$ tests. ${ }^{* *} p<0.01,{ }^{* * *} p<0.001$.

clinical relevance of isolated, increased $\mathrm{P}_{2} \mathrm{Y}_{1}$ activity is unclear since overall sensitivity to ADP was unaltered (-Fig. $\mathbf{3 B}$, - Table 2). Nonetheless, increased $P 2 Y_{1}$ receptor activity has been postulated to contribute to $\mathrm{P}_{2} \mathrm{Y}_{12}$ inhibitor resistance, 37,38 although evidence for this is lacking and to our knowledge no study has investigated the impact of hypoxia on $\mathrm{P}_{2} \mathrm{Y}_{12}$ inhibitor resistance.

An alternative explanation for the change in $R_{\max }$ with cangrelor is an increase in $\mathrm{P}_{2} \mathrm{Y}_{12}$ expression at altitude. Interestingly, a recent whole blood microarray study showed a twofold increase in $\mathrm{P}_{2} \mathrm{Y}_{12}$ expression in well acclimatised sojourners at high altitude. ${ }^{39}$ These findings were not validated in isolated platelets or by quantitative polymerase chain reaction. With either an increase in $\mathrm{P}_{2} \mathrm{Y}_{1}$ or $\mathrm{P}_{2} \mathrm{Y}_{12}$ expression, a change in sensitivity to ADP might be expected in the absence of inhibitors. However, as we detected no change in sensitivity to ADP, we propose that our finding of increased basal VASP phosphorylation is a compensatory mechanism that is also activated by hypoxia. Together with a lack of change in maximum phosphorylation induced by iloprost (-Fig. 5B) and a reduction in the percentage increase in phosphorylation induced by iloprost (-Fig. $\mathbf{5 C}$ ), this suggests that the basal ratio of VASP:VASP-P is altered by hypoxia. Although we did not observe reduced $\mathrm{P}_{2} \mathrm{Y}_{12}$ activity in response to ADP ( -Fig. 5A, C), we only studied a single high concentration of ADP $(5 \mu \mathrm{M})$, a concentration which induces near-maximal aggregation (-Fig. 3B). It may be that lower concentrations of ADP are unable to overcome increased basal VASP phosphorylation. If this is the case, it could represent a compensatory response to increased $\mathrm{P}_{2} \mathrm{Y}_{1}$ receptor activity or $\mathrm{P}_{2} \mathrm{Y}_{12}$ expression, the explanations proposed for the reduction in cangrelor efficacy. VASP-P is regulated by several mediators that are altered in hypoxic conditions. For example, exhaled nitric oxide (NO) levels increased over 48 hours in healthy subjects exposed acutely to $4,559 \mathrm{~m},{ }^{40}$ while raised circulating cyclic guanosine monophosphate (cGMP) levels and NO metabolites (nitrite and nitrate) were reported following gradual ascent to $5,200 \mathrm{~m} .{ }^{41} \mathrm{NO}$ inhibits platelet aggregation, at least in part via cGMP-mediated VASP phosphorylation as platelet adhesion to injured vessel walls could not be inhibited by NO in VASP-deficient mice. ${ }^{42}$ Interestingly, recent work demonstrated that nitrite led to VASP phosphorylation in isolated platelets in the presence of deoxygenated red blood cells. ${ }^{43}$ Thus, either increased NO synthase or nitrite reductase activity could explain our finding of increased basal VASP phosphorylation at altitude. ${ }^{44}$ - Fig. 6 summarises the proposed changes to purinergic signalling induced by hypoxia; however, this model will require further investigation to confirm its validity.

Kiouptsi et al examined the effects of brief exposure (30 minutes) of washed human platelets to extreme and moderate hypoxia ( 1 and $8 \%$ oxygen, respectively) on ex vivo expression of activated $\alpha_{\mathrm{II}} \beta_{3}$ (a key integrin in platelet aggregation $^{45}$ ) and aggregation. They reported that platelets exposed to extreme hypoxia had reduced expression of activated $\alpha_{\mathrm{IIb}} \beta_{3}$ in response to ADP stimulation. ${ }^{46}$ Our proposed model of hypoxia-induced alterations to purinergic signalling may offer a mechanistic explanation to these findings since an increase in VASP phosphorylation would attenuate $\mathrm{P}_{2} \mathrm{Y}_{12}$ activity and reduce ADP-induced activation of $\alpha_{\mathrm{II}} \beta_{3}{ }^{47-49}$ Kiouptsi et al also demonstrated that brief, extreme hypoxic exposure ( $1 \%$ oxygen, 30 minutes) reduced aggregation of hypoxic washed platelets in response to TRAP-6. However, sensitivity of PRP to TRAP- 6 amide was unchanged. ${ }^{46}$ Our data did not show any 


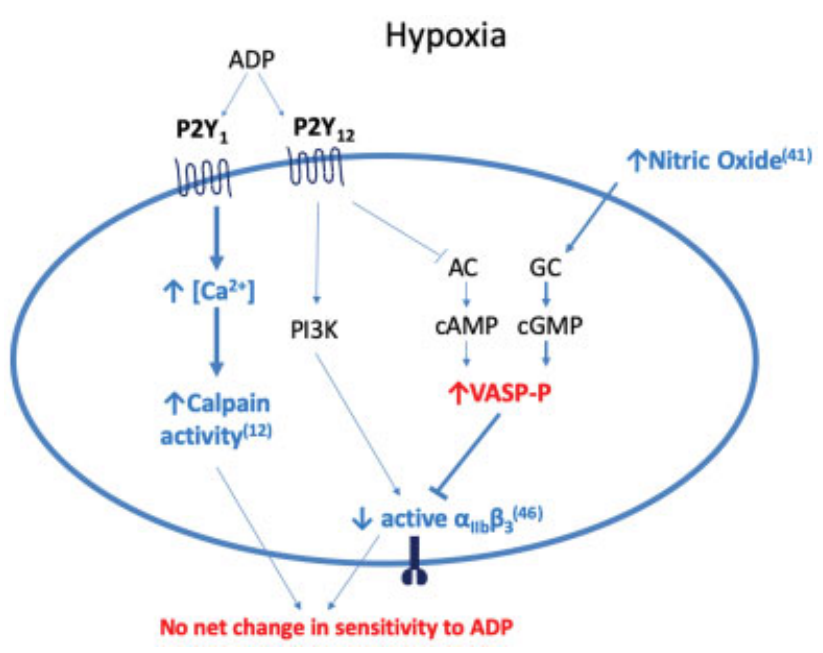

Fig. 6 Proposed model of hypoxia-induced changes to purinergic signalling. Our findings are in red, while those of other studies examining the impact of hypoxia on platelet signalling are in blue. Our data suggest that hypoxia up-regulates the basal level of vasodilatorstimulated phosphoprotein (VASP) phosphorylation, a key determinant of platelet aggregation downstream of the $\mathrm{P}_{2} \mathrm{Y}_{12}$ receptor. Other work demonstrating increased nitric oxide (NO) levels at altitude provides a possible mechanism for this finding. ${ }^{41}$ PhosphorylatedVASP (VASP-P) inhibits the expression of active $\alpha_{11 b} \beta_{3}$ expression, which links to Kiouptsi et al's finding that hypoxic platelets have lower expression of active $\alpha_{11 \mathrm{~b}} \beta_{3}$ in response to adenosine diphosphate (ADP). ${ }^{46}$ Increased P2Y $\mathrm{Y}_{1}$ pathway activity would be consistent with Tyagi et al's finding that calpains are up-regulated by hypoxia. ${ }^{12}$ Since we found no change in overall sensitivity to ADP, it may be that the changes produced by hypoxia in these two pathways counteract one another. AC, adenylate cyclase; ADP, adenosine diphosphate; CAMP, cyclic adenosine monophosphate; cGMP, cyclic guanine monophosphate; GC, guanylate cyclase; PI3K, phosphoinositide 3-kinase.

difference in TRAP-6 sensitivity on day 6 (-Fig. 3E), but reduced sensitivity was observed after a longer duration of hypoxia. It could be that hypoxia-induced changes in sensitivity to TRAP- 6 amide evolve over time, and only after 11 days was the effect on platelets pronounced enough to be seen in PRP. Interestingly, transcription of the receptor for TRAP- 6 amide, protease-activated receptor 1 , has also previously been shown to be down-regulated in hypoxic cancer cells, ${ }^{50}$ but this has not been investigated in platelets and would be an interesting topic for further research.

This study has several limitations. First, it is difficult to predict the clinical relevance of subtle changes to signalling when overall sensitivity to the agonist is not altered. Additionally, our study only examined the purinergic pathways in detail, and it is possible that other subtle differences exist in other signalling pathways. Finally, our study had a slight gender imbalance, and sex has been shown to affect platelet aggregation and purinergic signalling, ${ }^{51}$ implying our results may be more relevant to women than men.

Detailed investigation of purinergic signalling provided evidence of increased VASP phosphorylation and impaired inhibition of aggregation by a $\mathrm{P}_{2} \mathrm{Y}_{12}$ antagonist. We did not, however, find evidence of increased sensitivity to any platelet agonists despite the substantial evidence of hypoxia-induced platelet hyper-reactivity in the literature. However, the observed changes in aggregation in response to an ADP antagonist are of potential therapeutic significance to highaltitude sojourners and hypoxic sea-level patients prescribed platelet inhibitors and warrant further investigation.

\section{What is known about this topic?}

- Exposure to hypobaric hypoxia is thought to induce a thrombogenic phenotype, with increased risk of stroke in those residing at high altitude.

- The platelet proteome is altered by hypoxia and previous work has suggested platelet sensitivity to ADP is increased at altitude.

\section{What does this paper add?}

- Using optimul aggregometry, we show no significant changes in the dose-response of several platelet receptor agonists.

- Hypoxia suppressed the extent to which cangrelor inhibited platelet aggregation to ADP, a finding with potential implications for hypoxic patients taking platelet inhibitors.

\section{Authors' Contributions}

G.G.P., M.V.C., T.D.W. and A.A.R.T. conceived the study and designed the experiments; G.G.P., J.M.Y., J.A.W., C.J.G., R.C. D., E.W.L., G.S.T., M.V.C. and S.R.W. performed experiments and assisted with logistics; G.G.P., M.V.C., T.D.W., J. K.B. and A.A.R.T. analysed and interpreted the data; G.G.P. and A.A.R.T. drafted the manuscript with contributions from all authors.

\section{Funding}

This study received funding from the Wilderness Medical Society Charles S. Houston Grant, supported by the Academy of Wilderness Medicine. It also received funding from British Heart Foundation, (Grant/Award Number: 'FS/ 18/13/3328','PG/15/47/31591'), Wilderness Medicine Society (Grant/Award Number: 'Charles S. Houston grant') and Wellcome Trust, (Grant/Award Number: '103258/Z/ 13/Z,A').

\section{Conflict of Interest}

The authors declare no competing financial interests. A.A. R.T. reports non-financial support from Actelion UK Ltd, outside the submitted work.

\section{Acknowledgments}

We thank the APEX 5 expedition members for their participation. We thank the following companies for loan of equipment and technical support: Platelet Solutions Ltd., BMG LABTECH Ltd., Hettich Centrifuges, QInstruments, Genlab and Philip Harris Ltd. These companies had no role 
in the study design or interpretation of the results. We thank Dr. Zorka Castillo (Universidad Mayor de San Andrés, La Paz, Bolivia), Dr. Martin Waterfall (Flow Cytometry Facility, University of Edinburgh) and Dr. Craig Nash (University of Michigan) for technical support.J.K.B. acknowledges funding support from a Wellcome-Beit Intermediate Clinical Fellowship (103258/Z/13/Z,A), BBSRC Institute Strategic Program Grant and the UK Intensive Care Foundation. A.A.R.T. and M.C. acknowledge funding support from the British Heart Foundation (Intermediate Clinical Fellowship to A.A.R.T., FS/ 18/13/3328; Project Grant supporting M.C., PG/15/47/ 31591). S.R.W. acknowledges support from a Wellcome Trust Senior Clinical Fellowship (209220). G.G.P. acknowledges funding support from a British Society of Haemostasis and Thrombosis travel grant and the Principal's Go Abroad fund (University of Edinburgh). The expedition received funding from the following charitable organisations: The JABBS Foundation, Scottish Mountaineering Trust, Gilchrist Educational Trust and the Mount Everest Foundation.

\section{References}

1 Bärtsch P. How thrombogenic is hypoxia? JAMA 2006;295(19): 2297-2299

2 Anand AC, Jha SK, Saha A, Sharma V, Adya CM. Thrombosis as a complication of extended stay at high altitude. Natl Med J India 2001;14(04):197-201

3 Jha SK, Anand AC, Sharma V, Kumar N, Adya CM. Stroke at high altitude: Indian experience. High Alt Med Biol 2002;3(01):21-27

4 Schaber M, Leichtfried V, Fries D, et al. Influence of acute normobaric hypoxia on hemostasis in volunteers with and without acute mountain sickness. BioMed Res Int 2015;2015:593938

5 Toff WD, Jones CI, Ford I, et al. Effect of hypobaric hypoxia, simulating conditions during long-haul air travel, on coagulation, fibrinolysis, platelet function, and endothelial activation. JAMA 2006;295(19):2251-2261

6 Rocke AS, Paterson GG, Barber MT, et al. Thromboelastometry and platelet function during acclimatization to high altitude. Thromb Haemost 2018;118(01):63-71

7 Pichler Hefti J, Risch L, Hefti U, et al. Changes of coagulation parameters during high altitude expedition. Swiss Med Wkly 2010;140(7-8):111-117

8 Ninivaggi M, de Laat M, Lancé MMD, et al. Hypoxia induces a prothrombotic state independently of the physical activity. PLoS One 2015;10:e0141797

9 Nieswandt B, Pleines I, Bender M. Platelet adhesion and activation mechanisms in arterial thrombosis and ischaemic stroke. J Thromb Haemost 2011;9(Suppl 1):92-104

10 Jennings LK. Mechanisms of platelet activation: need for new strategies to protect against platelet-mediated atherothrombosis. Thromb Haemost 2009;102(02):248-257

11 Lehmann T, Mairbäurl H, Pleisch B, Maggiorini M, Bärtsch P, Reinhart WH. Platelet count and function at high altitude and in high-altitude pulmonary edema. J Appl Physiol (1985) 2006; 100(02):690-694

12 Tyagi T, Ahmad S, Gupta N, et al. Altered expression of platelet proteins and calpain activity mediate hypoxia-induced prothrombotic phenotype. Blood 2014;123(08):1250-1260

13 Fox JE, Taylor RG, Taffarel M, Boyles JK, Goll DE. Evidence that activation of platelet calpain is induced as a consequence of binding of adhesive ligand to the integrin, glycoprotein IIb-IIIa. J Cell Biol 1993;120(06):1501-1507

14 Jacobson KA, Deflorian F, Mishra S, Costanzi S. Pharmacochemistry of the platelet purinergic receptors. Purinergic Signal 2011;7 (03):305-324
15 Keyes LE, Mather L, Duke C, et al. Older age, chronic medical conditions and polypharmacy in Himalayan trekkers in Nepal: an epidemiologic survey and case series. J Travel Med 2016;23 (06):1-6

16 Chan MV, Warner TD. Standardised optical multichannel (optimul) platelet aggregometry using high-speed shaking and fixed time point readings. Platelets 2012;23(05):404-408

17 Butt E, Abel K, Krieger M, et al. cAMP- and cGMP-dependent protein kinase phosphorylation sites of the focal adhesion vasodilator-stimulated phosphoprotein (VASP) in vitro and in intact human platelets. J Biol Chem 1994;269(20):14509-14517

18 Hollopeter G, Jantzen H-M, Vincent D, et al. Identification of the platelet ADP receptor targeted by antithrombotic drugs. Nature 2001;409(6817):202-207

19 Schwarz UR, Geiger J, Walter U, Eigenthaler M. Flow cytometry analysis of intracellular VASP phosphorylation for the assessment of activating and inhibitory signal transduction pathways in human platelets-definition and detection of ticlopidine/clopidogrel effects. Thromb Haemost 1999;82(03):1145-1152

20 Glenn JR, Dovlatova N, White AE, Dhillon K, Heptinstall S, Fox SC. 'VASPFix' for measurement of VASP phosphorylation in platelets and for monitoring effects of P2Y12 antagonists. Thromb Haemost 2014;111(03):539-548

21 Belsley DA, Kuh E, Welsch RE. Detecting Influential Observations and Outliers. In: Regression Diagnostics: Identifying Influential Data and Sources of Collinearity. Hoboken: John Wiley \& Sons, Inc; 1980:6-84

22 Jones E, Oliphant T, Peterson P, et al. SciPy: open source scientific tools for Python [Internet]. 2001. Available at: http://www.scipy. org/. Accessed October 16, 2019

$23 \mathrm{R}$ Core Team. R: a language and environment for statistical computing. $\mathrm{R}$ Foundation for Statistical ComputingVienna, Austria [Internet]. 2019. Available at: http://www.r-project.org/. Accessed October 16, 2019

24 Würtz M, Hvas A-M, Kristensen SD, Grove EL. Platelet aggregation is dependent on platelet count in patients with coronary artery disease. Thromb Res 2012;129(01):56-61

25 Chan MV, Armstrong PCJ, Papalia F, Kirkby NS, Warner TD. Optical multichannel (optimul) platelet aggregometry in 96-well plates as an additional method of platelet reactivity testing. Platelets 2011;22(07):485-494

26 Würtz M, Hvas A-M, Christensen KH, Rubak P, Kristensen SD, Grove EL. Rapid evaluation of platelet function using the Multiplate ${ }^{\circledR}$ Analyzer. Platelets 2014;25(08):628-633

27 Beck F, Geiger J, Gambaryan S, et al. Temporal quantitative phosphoproteomics of ADP stimulation reveals novel central nodes in platelet activation and inhibition. Blood 2017;129(02): e1-e12

28 Nisar S, Kelly E, Cullen PJ, Mundell SJ. Regulation of P2Y1 receptor traffic by sorting Nexin 1 is retromer independent. Traffic 2010; 11(04):508-519

29 Magalhaes AC, Dunn H, Ferguson SS. Regulation of GPCR activity, trafficking and localization by GPCR-interacting proteins. Br J Pharmacol 2012;165(06):1717-1736

30 Jin J, Daniel JL, Kunapuli SP. Molecular basis for ADP-induced platelet activation. II. The P2Y1 receptor mediates ADP-induced intracellular calcium mobilization and shape change in platelets. J Biol Chem 1998;273(04):2030-2034

31 Grocott M, Montgomery H, Vercueil A. High-altitude physiology and pathophysiology: implications and relevance for intensive care medicine. Crit Care 2007;11(01):203

32 Feary JR, Rodrigues LC, Smith CJ, Hubbard RB, Gibson JE. Prevalence of major comorbidities in subjects with COPD and incidence of myocardial infarction and stroke: a comprehensive analysis using data from primary care. Thorax 2010;65(11):956-962

33 Marin JM, Carrizo SJ, Vicente E, Agusti AG. Long-term cardiovascular outcomes in men with obstructive sleep apnoea-hypopnoea with or without treatment with continuous positive airway pressure: an observational study. Lancet 2005;365(9464):1046-1053 
34 Sanner BM, Konermann M, Tepel M, Groetz J, Mummenhoff C, Zidek W. Platelet function in patients with obstructive sleep apnoea syndrome. Eur Respir J 2000;16(04):648-652

35 Maclay JD, McAllister DA, Johnston S, et al. Increased platelet activation in patients with stable and acute exacerbation of COPD. Thorax 2011;66(09):769-774

36 Bokinsky G, Miller M, Ault K, Husband P, Mitchell J. Spontaneous platelet activation and aggregation during obstructive sleep apnea and its response to therapy with nasal continuous positive airway pressure. A preliminary investigation. Chest 1995;108(03):625-630

37 Qureshi Z, Hobson AR. Clopidogrel "resistance": where are we now? Cardiovasc Ther 2013;31(01):3-11

38 Topçuoglu MA, Arsava EM, Ay H. Antiplatelet resistance in stroke. Expert Rev Neurother 2011;11(02):251-263

39 Jha PK, Sahu A, Prabhakar A, et al. Genome-wide expression analysis suggests hypoxia-triggered hyper-coagulation leading to venous thrombosis at high altitude. Thromb Haemost 2018; 118(07):1279-1295

40 Duplain H, Sartori C, Lepori M, et al. Exhaled nitric oxide in highaltitude pulmonary edema: role in the regulation of pulmonary vascular tone and evidence for a role against inflammation. Am J Respir Crit Care Med 2000;162(01):221-224

41 Levett DZ, Fernandez BO, Riley HL, et al; Caudwell Extreme Everest Research Group. The role of nitrogen oxides in human adaptation to hypoxia. Sci Rep 2011;1:109

42 Massberg S, Grüner S, Konrad I, et al. Enhanced in vivo platelet adhesion in vasodilator-stimulated phosphoprotein (VASP)-deficient mice. Blood 2004;103(01):136-142

43 Srihirun S, Piknova B, Sibmooh N, et al. Phosphorylated vasodilator-stimulated phosphoprotein (P-VASPSer239) in platelets is increased by nitrite and partially deoxygenated erythrocytes. PLoS One 2018;13:e0193747

44 Webb AJ, Milsom AB, Rathod KS, et al. Mechanisms underlying erythrocyte and endothelial nitrite reduction to nitric oxide in hypoxia: role for xanthine oxidoreductase and endothelial nitric oxide synthase. Circ Res 2008;103(09):957-964

45 Versteeg HH, Heemskerk JWM, Levi M, Reitsma PH. New fundamentals in hemostasis. Physiol Rev 2013;93(01):327-358

46 Kiouptsi K, Gambaryan S, Walter E, Walter U, Jurk K, Reinhardt C. Hypoxia impairs agonist-induced integrin $\alpha_{\mathrm{IIb}} \beta_{3}$ activation and platelet aggregation. Sci Rep 2017;7(01):7621

47 Kauffenstein G, Bergmeier W, Eckly A, et al. The P2Y(12) receptor induces platelet aggregation through weak activation of the $\alpha(\mathrm{IIb})$ $\beta$ (3) integrin-a phosphoinositide 3-kinase-dependent mechanism. FEBS Lett 2001;505(02):281-290

48 Kamae T, Shiraga M, Kashiwagi H, et al. Critical role of ADP interaction with P2Y12 receptor in the maintenance of alpha (IIb)beta3 activation: association with Rap1B activation.J Thromb Haemost 2006;4(06):1379-1387

49 Horstrup K, Jablonka B, Hönig-Liedl P, Just M, Kochsiek K, Walter U. Phosphorylation of focal adhesion vasodilator-stimulated phosphoprotein at Ser157 in intact human platelets correlates with fibrinogen receptor inhibition. Eur J Biochem 1994;225(01): $21-27$

50 Caradec J, Sirab N, Keumeugni C, et al. 'Desperate house genes': the dramatic example of hypoxia. Br J Cancer 2010;102(06): 1037-1043

51 Becker DM, Segal J, Vaidya D, et al. Sex differences in platelet reactivity and response to low-dose aspirin therapy. JAMA 2006; 295(12):1420-1427 